\title{
IV. Ventilación no invasiva en pacientes con enfermedades neuromusculares
}

\author{
CÉSAR MAQUILÓN O.*, SERGIO CASTILLO**, GUILLERMO MONTIEL***, \\ GERARDO N. FERRERO R.****, ADA TOLEDO***** y CATALINA SIROTI******
}

\section{Noninvasive ventilation in patients with neuromuscular diseases}

El compromiso funcional respiratorio en las enfermedades neuromusculares (ENM) se manifiesta por hipoventilación alveolar (hipoxemia e hipercapnia) debido a una disminución de la actividad de la bomba muscular ventilatoria, con pulmones estructuralmente sanos. El compromiso de la bomba muscular respiratoria puede ser ocasionado por una pérdida del estímulo del centro respiratorio, anomalías en la conducción del estímulo o enfermedades propias del músculo (Figura 1).

Las enfermedades neuromusculares pueden ser de evolución lenta o estables, como en la secuela de polio o lesiones traumáticas de la médula espinal y algunas enfermedades del centro respiratorio, intermitente, como ocurre en la miastenia gravis, intermedia, como acontece en las enfermedades de los músculos respiratorios, o rápidas como en la esclerosis lateral amiotrófica (ELA) y ciertas patología de los nervios periféricos (Tabla 1) 1,2.

El centro respiratorio modula las aferencias estimuladoras e inhibidoras que provienen de los quimioreceptores centrales y periféricos, mecanoreceptores, centro cerebrales superiores, etc. El estímulo viaja hacia los músculos inspiratorios a través del sistema nervioso central y finalmente por los nervios periféricos (Figura 1). En cada uno de estos niveles pueden ocurrir altera- ciones que modifiquen la actividad de la bomba muscular y producir grados variables de hipoxemia e hipoventilación alveolar.

La edad de aparición del problema respiratorio depende de la patología de base, pero por regla general las miopatías (de evolución intermedia) aparecen en la infancia, permitiendo cierta adaptación a la disfunción muscular progresiva (Tabla 1)3. En cambio, la esclerosis lateral amiotrófica ocurre en edades más avanzadas, que sumado a la rapidez de la evolución, determinan una pobre adaptación del paciente y los familiares a la discapacidad que produce esta enfermedad.

La ventilación no invasiva debe ser considerada como la primera herramienta terapéutica en la insuficiencia ventilatoria del paciente neuromuscular sin compromiso bulbar asociado ${ }^{1-3}$. Las técnicas de VNI están destinadas a pacientes que conservan la actividad del centro respiratorio, pero que son incapaces de mantener una ventilación espontánea adecuada a las demandas metabólicas. La presión (Pmi) que deben generar los músculos inspiratorios (MI) para generar un determinado volumen corriente $(\mathrm{Vt})$, está en relación directa con el $\mathrm{Vt}$, la elastancia $(\mathrm{E})$ tóracopulmonar, el flujo (FVA) y la resistencia (RVA) de la vía aérea (RVA) y la PEEP intrínseca o auto-PEEP.

\footnotetext{
* Residente, Unidad de Cuidados Intermedios, Instituto Nacional del Tórax. Encargado del Programa AVNIA, Ministerio de Salud, Chile.

** Neurólogo, Servicio de Neurología, Hospital del Salvador.

*** Neumólogo, Servicio de Laboratorio de Sueño, Oxigenoterapia y Ventilación no invasiva, Hospital Antonio A. Cetrangolo, Buenos Aires, Argentina.

**** Lic. Kinesiólogo, Hospital de Rehabilitación Respiratoria María Ferrer, Buenos Aires, Argentina. Fellow Internacional de la Asociación Americana de Cuidados Respiratorios (AARC).

***** Neumóloga, Unidad de Cuidados Intensivos y Laboratorio de Sueño, Hospital de Rehabilitación Respiratoria María Ferrer, Buenos Aires, Argentina.

******Lic. Kinesióloga, Unidad de Terapia Intensiva, Hospital Naval Pedro Mallo y Servicio de Laboratorio de Sueño, Oxigenoterapia y Ventilación no invasiva, Hospital Antonio A. Cetrangolo, Buenos Aires, Argentina.
} 


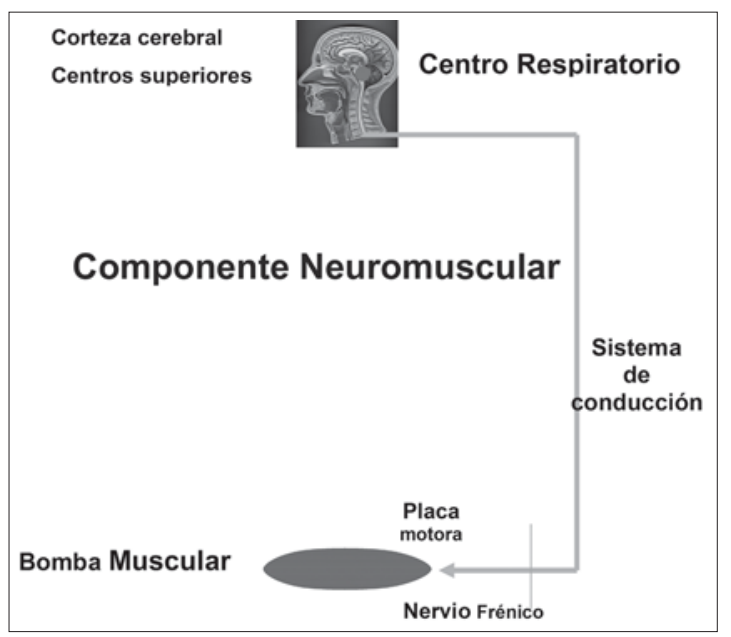

Figura 1. Componentes del estímulo respiratorio y bomba muscular.

$\begin{array}{cl}\text { Pmi }= & (\mathrm{E} \cdot \mathrm{Vt})+(\mathrm{RVA} \cdot \mathrm{FVA})+ \\ \frac{\text { Auto-PEEP }}{(\text { Componente }} & \frac{(\text { Componente de carga })}{\text { neuromuscular })}\end{array}$

Por lo tanto, podemos separar los dos componentes de la ecuación en:

1. Componente Neuromuscular.

- Generación del estímulo

- Conducción y transmisión del estímulo

- Bomba muscular

2. Carga contra la cual se deben contraer los músculos inspiratorios.

- Elastancia

- Conductancia

Conocer los mecanismos que pueden alterar el funcionamiento del sistema neuromuscular y la carga contra la cual se deben contraer los MI, es de utilidad para programar correctamente los parámetros del respirador ${ }^{4-6}$.

\section{Enfermedades de los músculos respiratorios}

Las enfermedades neuromusculares evolucionan generalmente en forma lenta y progresiva ${ }^{3}$. La presencia de complicaciones respiratorias habitualmente son tardías en el curso de la evolución de la enfermedad. Los primeros síntomas van a estar en relación con el sueño (somnolencia diurna excesiva, cefalea matinal) y la disnea que se manifiesta durante la actividad física. Cuando existe compromiso diafragmático, puede evidenciarse ortopnea o movimiento paradojal del abdomen. La aparición de cianosis, eritro-
Tabla 1. Enfermedades neuromusculares que pueden requerir ventilación no invasiva. Clasificación según velocidad de progresión de la enfermedad

1. Estables o lentamente progresivas
a) Injuria médula espinal con tetraplejia (nivel C5)
b) Secuela de poliomielitis
c) Parálisis diafragmática
d) Atrofia muscular espinal II y III

2. Progresivas (años o décadas)
a) Miopatías congénitas
b) Miopatías metabólicas
c) Distrofia muscular de Duchenne

3. Rápidamente progresivas

a) Esclerosis lateral amiotrófica

citosis, hipertensión pulmonar, insuficiencia cardíaca derecha y severa incapacidad física, pueden ocurrir en los estados avanzados no tratados.

Los pacientes con debilidad muscular respiratoria presentan un patrón de función pulmonar de tipo restrictivo con compromiso de los volúmenes pulmonares ${ }^{3}$. La capacidad vital (CV) disminuye entre un 40 y $70 \%$. También se observan reducciones en la capacidad pulmonar total (CPT) y la capacidad residual funcional (CRF), a expensas del volumen de reserva espiratoria. El volumen residual (VR) está relativamente conservado o levemente aumentado, y la relación $\mathrm{VEF}_{1} / \mathrm{CV}$ se mantiene en rangos normales.

Se produce una disminución progresiva de la distensibilidad pulmonar y de la pared torácica ${ }^{3}$. La causa de la misma se desconoce, pero probablemente se relaciona a la presencia de microatelectasias difusas (observadas en autopsias). La distensibilidad de la pared torácica está disminuida aproximadamente un $70 \%$ del valor normal, debido a un incremento en la rigidez de la parrilla costal, que puede ser causada por el endurecimiento de los tendones y ligamentos adheridos a la parrilla costal, y la anquilosis de las articulaciones costoesternales y tóracovertebrales. En pacientes con cuadriplejía, el desarrollo de espasticidad y fibrosis de los músculos intercostales puede empeorar la rigidez de la pared torácica.

El patrón respiratorio en estos pacientes se caracteriza por una ventilación rápida y superficial con suspiros infrecuentes ${ }^{3}$. La taquipnea podría ser causada por estímulos provenientes de los músculos respiratorios debilitados, recepto- 
res intrapulmonares, o reducción de la distensibilidad del sistema respiratorio. El intercambio gaseoso se mantiene estable, con un gradiente alvéolo-arterial de oxígeno relativamente normal. La presión parcial de $\mathrm{CO}_{2}$ arterial puede estar reducida al comienzo de la enfermedad, pero se eleva cuando la fuerza muscular respiratoria cae por debajo de un $30 \%$ del valor predictivo. De acuerdo a la entidad nosológica, la respuesta ventilatoria a la hipercapnia, se encuentra normal o disminuida.

La presión inspiratoria máxima (PIMax) y la presión espiratoria máxima (PEMax) se encuentran disminuidas, la primera en parte debido a la debilidad muscular y a la inestabilidad de los músculos de la pared torácica ${ }^{2,3}$. La PEMax se encuentra a menudo más comprometida que la PIMax, debido a que los músculos respiratorios se mantienen entrenados con cada respiración, mientras que la espiración es habitualmente pasiva. Además, la incapacidad de poder inspirar hasta CPT lleva a una subestimación de la PEMax. La debilidad de los músculos espiratorios ocasiona una disminución de la efectividad del reflejo de la tos, dado por una caída en el flujo espiratorio tusígeno máximo (FETM).

La capacidad residual funcional (CRF) sufre cambios en la posición de decúbito, cayendo un $20 \%$ de la posición erecta al decúbito supino (600 a $800 \mathrm{~mL}$ ). Esto es debido a cambios en la posición del diafragma. El diafragma se ubica en una posición más cefálica en decúbito supino, debido a la presión del contenido abdominal, actuando como fuerza espiratoria, reduciendo así el volumen pulmonar. La reducción de la CRF es a expensas de una caída principalmente del volumen de reserva espiratoria. En adición, la sangre de las extremidades se desplaza hacia el tórax en decúbito dorsal, ocasionando una mayor caída de la CRF de alrededor de $300 \mathrm{~mL}$.

\section{Evaluación de los enfermos neuromusculares}

En general, los pacientes con enfermedades neuromusculares deben ser sometidos a pruebas funcionales respiratorias anualmente (Tabla $2)^{7,8}$. La frecuencia de estos controles debe aumentar cuando la capacidad vital y la PIMax disminuyen por debajo del $50 \%$ de su valor teórico o la $\mathrm{CV}$ es menor a $1,5 \mathrm{~L}$.

1. Capacidad vital: Evidencia la situación actual de la función respiratoria en el paciente. Es importante, en caso de contar con la misma, conocer cual es la CVF que maneja habitualmente dicho paciente. Se debe realizar en posición erguida y en decúbito dorsal (Figura 2).

2. Presión inspiratoria máxima: Representa la máxima presión que pueden generar los músculos inspiratorios a flujo cero. La medición se realiza con un manómetro desde volumen residual. En individuos normales la presión generada oscila entre 120-180 $\mathrm{cm} \mathrm{H}_{2} \mathrm{O}$, de acuerdo a la edad, género y altura.

3. Presión espiratoria máxima: Representa la máxima presión que pueden generar los músculos espiratorios a flujo cero. La medición se realiza con un manómetro desde capacidad pulmonar total. En individuos normales la presión generada oscila entre $150-200 \mathrm{~cm} \mathrm{H}_{2} \mathrm{O}$, de acuerdo a la edad, género y altura. La PEMax es el indicador más sensitivo para mensurar la capacidad tusígena en los pacientes neuromusculares. Una PEMax por debajo de $40 \mathrm{~cm}$ de $\mathrm{H}_{2} \mathrm{O}$ indica incapacidad de remover las secreciones del tracto respiratorio superior, con retención concomitante de las mismas e infección sobreagregada.

4. Flujo espiratorio tusígeno máximo: $\mathrm{Se}$ trata del flujo espiratorio máximo que puede generar un paciente desde CPT. El flujo tusígeno

Tabla 2. Compromiso respiratorio en las enfermedades neuromusculares

Presiones bucales máximas

PIMax $<20 \mathrm{~cm} \mathrm{H}_{2} \mathrm{O}$

PEMax $<40 \mathrm{~cm} \mathrm{H}_{2} \mathrm{O}$

Capacidad vital

$<30 \mathrm{~mL} / \mathrm{kg}$

$<20 \mathrm{~mL} / \mathrm{kg}$

$<10 \mathrm{~mL} / \mathrm{kg}$

Compromiso bulbar (debilidad o parálisis)

Gases arteriales

Hipercapnia

Hipoxemia
Incapacidad para ventilar adecuadamente

Incapacidad para toser y aclaramiento de secreciones

Incapacidad para toser adecuadamente Incapacidad para suspirar o prevención de atelectasias Incapacidad para ventilar adecuadamente Incapacidad para proteger la vía aérea y evitar aspiraciones

Incapacidad para ventilar adecuadamente Incapacidad para oxigenar adecuadamente 


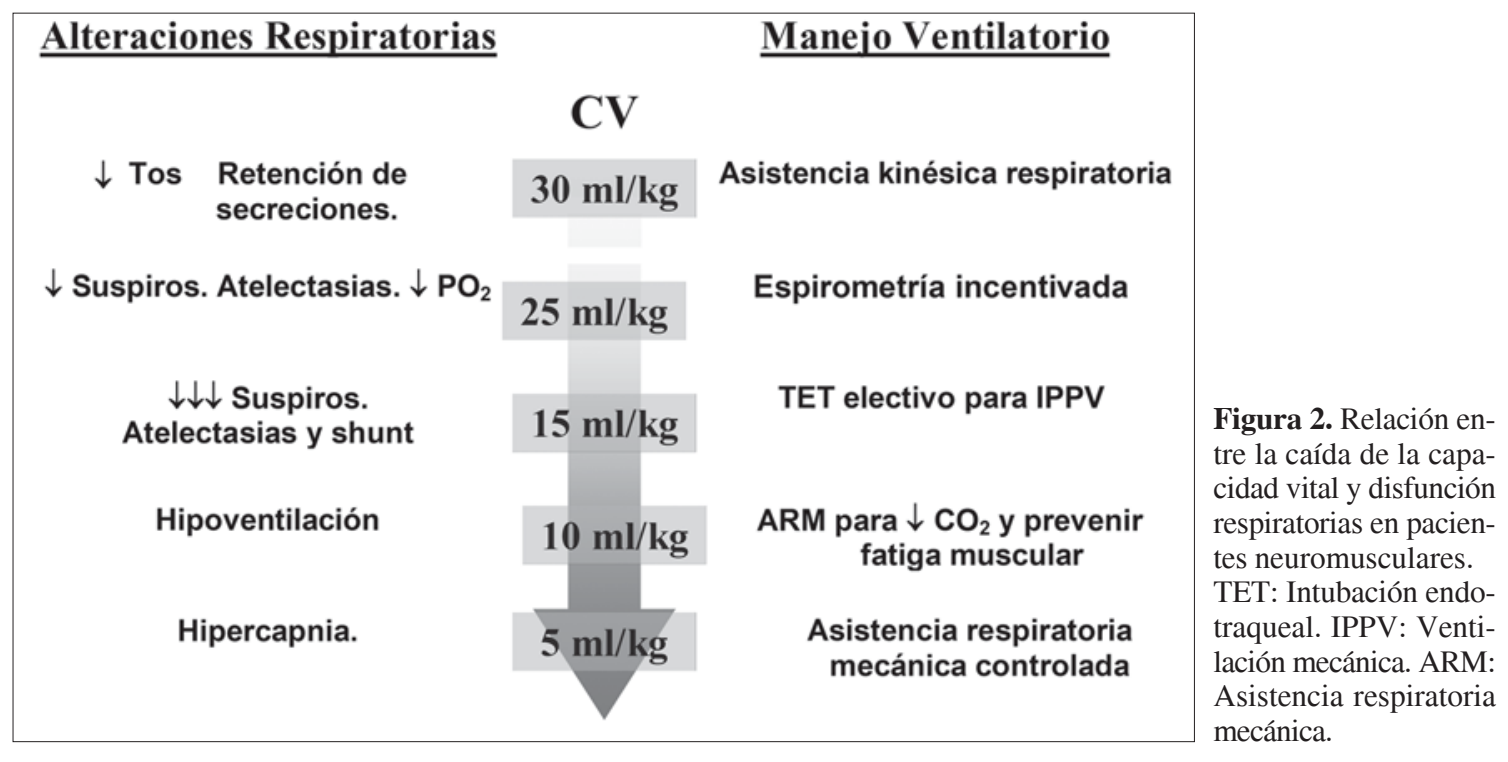

máximo pueden superar los 6 a $12 \mathrm{~L} / \mathrm{s}$ dependiendo del género, edad y altura. Los FETM menores a $160 \mathrm{~L} / \mathrm{min}$ indican incapacidad de mantener flujos tusígenos mínimos indispensables para la remoción de las secreciones bronquiales en el tracto respiratorio superior.

5. Gases en sangre: La hipoventilación alveolar $\left(\mathrm{PaCO}_{2}>45 \mathrm{mmHg}\right)$ se asocia a la debilidad de la bomba muscular inspiratoria y suele ser una manifestación tardía de la enfermedad neuromuscular ${ }^{9,10}$. La presencia de hipoxemia con $\mathrm{FiO}_{2}$ de 0,21 con un gradiente alvéolo-capilar aumentado nos indica déficit en el mecanismo intercambiador de gases, debido a un compromiso parenquimatoso (atelectasia, infiltrados, trastornos V/Q, etc). Aumento del exceso de base por encima de $4 \mathrm{mEq} / \mathrm{L}$ es predictor de falla ventilatoria y la alcalosis metabólica diurna puede ser el primer indicio de hipoventilación nocturna.

6. Polisomnografía y/u oximetría nocturna: Las manifestaciones más tempranas de la enfermedad ocurren durante el estadio REM del sueño, donde existe una importante hipotonía de los músculos de la faringe, de la caja torácica y de los músculos inspiratorios, permitiendo el desarrollo de hipoventilación alveolar y de importantes desaturaciones, evidenciadas en la oximetría de pulso. Estos episodios causan repetidos microdespertares con mala calidad del sueño, siendo comunes durante el día las cefaleas matinales, hipersomnolencia, cambios en el humor, disminución de la capacidad de concentración.

7. Pruebas de ejercicio: Las distintas pruebas de ejercicio (caminata a su propio paso, test de ejercicio incremental, etc), monitorizados con un oxímetro de pulso con memoria, permite realizar un seguimiento objetivable de la función cardiopulmonar durante la actividad física.

En la Figura 2 se describe la utilidad de la medición de la capacidad vital (CV) en el manejo de pacientes con Síndrome de Guillain Barré . Los pacientes que presentan una $\mathrm{CV}$ menor a $15 \mathrm{~mL} / \mathrm{kg}$ deben empezar con algún tipo de asistencia ventilatoria, sea VNI o presión positiva a través de tubo endotraqueal, según la experiencia del equipo médico tratante. Los controles deben hacerse cada cuatro horas como mínimo. De esa manera se puede anticipar el tratamiento con apoyo ventilatorio, evitando tratar a los paciente en estadios más avanzados, cuando la hipercapnia, acidosis y alteraciones cardiovasculares son más severas y difíciles de revertir. $\mathrm{Si}$ bien este esquema está relacionado con el manejo agudo de los pacientes con Guillain Barré, se puede aplicar este mismo criterio para controlar a los pacientes con otras enfermedades neuromusculares con deterioro progresivo. El mismo concepto se utiliza para el monitoreo de la presión inspiratoria máxima u otras medidas de fuerza o resistencia para evaluar a los músculos inspiratorios.

\section{Selección de pacientes con enfermedades neuromusculares candidatos a VNI}

El inicio de la VNI está indicado en pacientes neuromusculares con síntomas o signos rela- 
cionados con hipoventilación alveolar y alguna de las siguientes condiciones ${ }^{11-16}$ :

1. Hipercapnia diurna $\left(\mathrm{PaCO}_{2}>45 \mathrm{mmHg}\right)$ evidenciada por medio de la obtención de gases arteriales. La presencia de alcalosis metabólica puede ser un indicador de hipoventilación nocturna.

2. Desaturación durante el sueño, puede ocurrir sin hipercapnia diurna. La VNI se indica cuando existe una $\mathrm{SpO}_{2}$ menor de $89 \%$ durante por lo menos cinco minutos consecutivos o más del $10 \%$ del tiempo total monitorizado. La medición del flujo nasal y esfuerzo toracoabdominal es útil para descartar apneas obstructivas.

3. Rápido deterioro de la función respiratoria, con frecuentes consultas a unidades de emergencia por insuficiencia respiratoria, en pacientes con $\mathrm{CV}<50 \%$.

4. En pacientes que recibieron asistencia mecánica a través de tubo endotraqueal como consecuencia de infecciones pulmonares o por imposibilidad de eliminar el exceso de secreciones bronquiales durante los procesos agudos.

Si bien existe consenso de iniciar la VNI cuando se ha desarrollado hipercapnia diurna, actualmente se observa una tendencia a iniciar la VNI en períodos más tempranos, sobre todo en pacientes portadores de la enfermedad de Duchenne, cuando se constata hipoventilación nocturna ${ }^{11,12,17}$.

\section{Objetivos de la VNI en pacientes con enfermedades neuromusculares}

1. El objetivo primario es mejorar la ventilación alveolar y la oxigenación. Para ello se debe iniciar la VNI con $\mathrm{FiO}_{2}$ ambiental y se debe evitar el empleo de $\mathrm{FiO}_{2}$ elevadas para el mantenimiento de una oximetría "adecuada", ya que el aumento del oxígeno suministrado favorece la retención de $\mathrm{PaCO}_{2}$. El suplemento de oxígeno puede ser útil es caso de patología pulmonar agregada.

2. La incapacidad en el manejo de las secreciones bronquiales implica un abordaje kinésico agresivo, de lo cual dependerá en gran medida el poder evitar la invasión de la vía aérea superior con un tubo endotraqueal por mal manejo de las mismas ${ }^{13,18}$.

3. Mejorar la distensibilidad pulmonar, la aplicación de PEEP/EPAP permite aumentar la $\mathrm{CRF}$, disminuyendo las microatelectasias y mejorando el intercambio gaseoso ${ }^{15,16}$.
4. Mejorar la calidad del sueño. La VNI nocturna, al evitar los episodios de hipoventilación (centrales y obstructivos) mejora la arquitectura del sueño y disminuye los despertares.

5. Mejorar la calidad de vida y la supervivencia.

\section{Implementación de la ventilación no invasiva}

\section{Pacientes estables con insuficiencia respiratoria crónica}

Se puede iniciar en el domicilio, en el hospital o en el laboratorio de sueño ${ }^{1,10,19}$. Una vez que se decide iniciar la VNI, se debe preparar al paciente, brindarle contención emocional, explicarle el procedimiento, posición en decúbito a $45^{\circ}$, que esté lo más cómodo posible. Los pacientes deben tener una función bulbar adecuada, el mecanismo de la tos conservado, idealmente con pocas secreciones bronquiales y sin compromiso hemodinámico.

Se debe elegir una máscara nasal/facial adecuada a la cara/nariz del paciente y según la experiencia del operador ${ }^{1,10}$. Los respiradores volumétricos son muy utilizados en el manejo de este tipo de falla ventilatoria. En la actualidad, existe una tendencia a ventilar por presión, gatillado por flujo, con la posibilidad de agregar fácilmente presión positiva al final de la espiración, modificar el flujo inicial (rise time) y programar el tiempo inspiratorio (ti). Los modos de presión más usados son la presión de soporte (PS) y la presión controlada (PC). En los pacientes neuromusculares el agregado de una frecuencia respiratoria base es útil para controlar los episodios de apneas centrales. En caso de presentar respiración periódica los equipos de BiPAP automáticos son de extrema utilidad.

Cuando existe variaciones importantes de las resistencias toracopulmonares (cambios de decúbito, aumento de resistencia de la vía aérea superior), para evitar variaciones importantes del volumen corriente entregado, se debe emplear un BiPAP con volumen corriente asegurado con presión de soporte (AVAPS), que permite que los equipos de presión actúen en forma parecida a los respiradores volumétricos, pero agregando todo el confort y tecnología de los respiradores de turbina.

El objetivo inicial es lograr una adaptación diurna al procedimiento durante algunos días ${ }^{1,10}$. Cuando el paciente está adaptado se realizarán los ajustes finales durante la noche, tratando de alcanzar los objetivos de reposo de los músculos inspiratorios y mejoría del intercambio ga- 
seoso. Se debe constatar la eficacia de la VNI mediante polisomnografía u oximetría nocturna (cuando recibe VNI respirando aire ambiente) y realizar un seguimiento estricto durante los primeros meses.

El nivel ideal de $\mathrm{PaCO}_{2}$ no ha sido establecido, si bien alcanzar $40 \mathrm{mmHg}$ es una condición deseable, se pueden aceptar valores más elevados si los síntomas de hipoventilación están controlados y no existen evidencias de falla cardíaca derecha.

\section{Pacientes con insuficiencia respiratoria aguda}

Enfermos en los cuales la ventilación no invasiva se inició durante un episodio de insuficiencia respiratoria aguda y que, a pesar de estar médicamente estables, no recuperan la capacidad de respirar espontáneamente (ventilación obligada $)^{1,10}$. Estos pacientes generalmente son remitidos desde la unidad de cuidados intensivos. En general la mayoría están ventilados a través de una traqueostomía. Los objetivos de la terapia respiratoria en este grupo de pacientes se centran en prepararlos para retornar a la comunidad, instruir a sus cuidadores sobre cómo proveer el cuidado y, por último, educarlos para que sean capaces de participar en actividades recreativas y sociales. En este grupo de pacientes, el sistema de ventilación necesita evaluarse constantemente porque las necesidades del enfermo pueden cambiar día a día y hay que evaluar la posibilidad de retirar la traqueostomía (Tabla 3$)^{6}$.

\section{Contraindicaciones para realizar VNI en pacientes con enfermedades neuromusculares}

La única contraindicación absoluta para la VNI es la incapacidad para tolerar la máscara o el procedimiento $^{1,10}$. Por tal motivo, es fundamen-

Tabla 3. Criterios de decanulación en pacientes con enfermedades neuromusculares

1. Enfermo mentalmente alerta y cooperador

2. Necesidad mínima de oxígeno

3. Saturación arterial de oxígeno mayor a $90 \%$

4. Función bulbar conservada con mecanismo de la deglución intacto

5. Ausencia de historia de adicción a drogas o de ataques convulsivos

6. Flujo espiratorio máximo de la tos mayor de $3 \mathrm{~L} / \mathrm{s}$

7. Estructuras faciales intactas tal tener el equipamiento adecuado y contar con suficiente experiencia para desarrollar este tipo de tratamiento. Las contraindicaciones relativas también dependen de la experiencia del equipo tratante y se enumeran a continuación:

1. Compromiso severo de los músculos bulbares y función deglutoria alterada.

2. Falta de cooperación o motivación por parte del paciente o de los familiares.

3. Dificultad para el manejo de las secreciones bronquiales.

4. Requerimiento de VNI por más de 16 h/día.

\section{VNI domiciliaria en pacientes neuromusculares y con cifoescoliosis}

La información sobre los resultados de la VNI domiciliaria en pacientes con enfermedades neuromusculares se obtiene de trabajos prospectivos y descriptivos recientes, haremos referencia a un análisis de la literatura del año 1999.

Nauffal $^{19}$, describe el uso de VNI en 35 pacientes con cifoescoliosis y 27 pacientes con enfermedades neuromusculares seguidos durante 18 meses, los pacientes mejoraron sus índices de calidad de vida, redujeron las tasas de admisión hospitalaria y mejoraron significativamente sus gases arteriales. Mellies y cols ${ }^{20}$, examinaron durante un período promedio de 25 meses el efecto de la VNI en 38 niños con enfermedades neuromusculares progresivas, con edad promedio de 12,3 \pm 4,1 años (rango: 619). Los niños fueron sometidos a una evaluación sistemática y regular que incluía estudios de sueño, evaluación funcional y gasometría arterial. La VNI fue bien tolerada y altamente efectiva en revertir los desórdenes respiratorios durante el sueño y la falla respiratoria crónica que presentaban antes del inicio de este apoyo ventilatorio. Janssens y cols ${ }^{21}$, publicaron en el 2003 los resultados del seguimiento por siete años de 40 pacientes neuromusculares y 19 pacientes con cifoescoliosis e hipoventilación diurna y reportaron mejoría en los gases arteriales, índices de calidad de vida y reducción en el número de hospitalizaciones por año. Los pacientes tiene una adherencia a la VNI mayor de $90 \%$ y la curva de Kapplan Meier demuestra que los pacientes cifoescolióticos tienen una sobrevida de $84 \%$ a los 7 años.

En 1999, Annane y cols ${ }^{22}$, reportan el resultado del análisis de cuatro ensayos elegibles que incluyeron 51 pacientes con afecciones neuromusculares o de la pared torácica, el tratamiento con VNI favoreció la reducción de los nive- 
les de hipercapnia, la mejoría de la saturación promedio nocturna y el riesgo de morir en el año siguiente a la administración de la ventilación mecánica nocturna (-0,259; IC 95\%: -0,478 a -0,041). Sin embargo, el análisis concluye que las pruebas actuales sobre el beneficio terapéutico de la ventilación mecánica no invasiva son débiles, pero coherentes, y sugieren el alivio de los síntomas de la hipoventilación crónica a corto plazo. En dos pequeños estudios la supervivencia se prolongó con la aplicación de VNI.

\section{Recomendaciones}

La ventilación mecánica debe ofrecerse como una opción terapéutica a los pacientes con hipoventilación crónica debida a enfermedades neuromusculares. Se necesitan ensayos aleatorios adicionales con un número mayor de pacientes para confirmar los efectos beneficiosos a largo plazo de la ventilación mecánica nocturna sobre la calidad de vida, la morbilidad y mortalidad, para evaluar la proporción costo/beneficio y comparar los diferentes tipos y modalidades de ventilación mecánica.

\section{Bibliografía}

1.- Noninvasive ventilation: principles and applications, edited by Nicholas Hill. 2001. Published by Futura Publishing Company.

2.- LISBOA C, DÍAZ O, FADIC R. Ventilación no invasiva en pacientes con enfermedades neuromusculares y en pacientes con alteraciones de la caja torácica. Arch Bronconeumol 2003; 39: 314-20.

3.- Respiratory Dysfunctions in Neuromuscular Disease. Edited by Fanburg B. and Sicilian L. Clinics in Chest Medicine 1994, Volume 15 number 4.

4.- PURRO A, APPENDINI L, D E GAETANO A, GUDJONSDOTTIR M, DONNER C F, ROSSI A. Physiologic determinants of ventilator dependence in long-term mechanically ventilated patients. Am J Respir Crit Care Med 2000; 161: 1115-23.

5.- BELLEMARE F, GRASSINO A. Effect of pressure and timing of contraction on human diaphragm fatigue. J Appl Physiol 1982; 53: 1190-5.

6.- GOODENBERGER D M, COUSER J I JR, MAY J J. Successful discontinuation of ventilation via tracheostomy by substitution of nasal positive pressure ventilation. Chest 1992; 102: 1277-9.

7.- VIROSLAV J, ROSENBLATT R, TOMAZEVIC S M. Respiratory management, survival, and quality of life for high-level traumatic tetraplegics. Respir Care Clin N Am 1996; 2: 313-22.
8.- BACH J R. Conventional approaches to managing neuromuscular ventilatory failure. In Bach JR, ed Pulmonary Rehabilitation: the obstructive and paralytic conditions. Philadelphia: Hanly and Belfus, page 285-301,1996.

9.- WARD S, CHATWIN M, HEATHER A, SIMONDS A. Randomised controlled trial of non-invasive ventilation for nocturnal hypoventilation in neuromuscular and chest wall disease patients with daytime normocapnia. Thorax 2005; 60: 1019-24.

10.- Consensus Conference. Clinical indications for NIV in chronic respiratory failure due to restrictive lung disease, COPD and nocturnal hypoventilation. Chest 1999; 116: 521-34.

11.- MASA J F, CELLI B R, RIESCO J A, SÁNCHEZ DE COS J, DISDIER C, SOJO A. Noninvasive positive pressure ventilation and not oxygen may prevent overt ventilatory failure in patients with chest wall diseases. Chest 1997; 112: 207-13.

12.- BACH J R, ISHIKAWA Y, KIM H. Prevention of pulmonary morbidity for patients with Duchenne muscular dystrophy. Chest 1997; 112: 1024-8.

13.- BACH J R. Mechanical insufflation-exsufflation. Comparison of peak expiratory flows with manually assisted and unassisted coughing techniques. Chest 1993; 104: 1553-62.

14.- BACH J R, ALBA A S. Noninvasive options for ventilatory support of the traumatic high level quadriplegic patient. Chest 1990; 98: 613-9.

15.- BACH J R. New approaches in the rehabilitation of the traumatic high level quadriplegic. Am J Phys Med Rehabil 1991; 70: 13-9.

16.- BACH J R. Amyotrophic lateral sclerosis: prolongation of life by noninvasive respiratory aids. Chest 2002; 122:92-8.

17.- Tratado de Neumología Albert Richard. Trastornos Neuromusculares Agudos y Crónicos. Capitulo 72, 2001.

18.- AVENDAÑO M, GÜELL R. Rehabilitación en pacientes con enfermedades neuromusculares y con deformidades de la caja torácica. Arch Bronconeumol 2003; 39:559-65.

19.- NAUFFAL D, DOMÉNECH R, MARTÍNEZ GARCÍA M A, COMPTE L, MACIÁN V, PERPIÑÁ M. Noninvasive positive pressure home ventilation in restrictive disorders: outcome and impact on healthrelated quality of life. Respir Med 2002; 96: 777-83.

20.- MELLIES U, RAGETTE R, DOHNA SCHWAKE C, BOEHM H, VOIT T, TESCHLER H. Long-term noninvasive ventilation in children and adolescents with neuromuscular disorders. Eur Respir J 2003; 22: 631-6.

21.- JANSSENS J P, DERIVAZ S, BREITENSTEIN E, DE MURALT B, FITTING J W, CHEVROLET J C, et al. Changing patterns in long-term noninvasive ventilation: a 7-year prospective study in the Geneva Lake area. Chest 2003; 123: 67-79.

22.- ANNANE D, ORLIKOWSKI D, CHEVRET S, CHEVROLET J C, RAPHAËL J C. Nocturnal mechanical ventilation for chronic hypoventilation in patients with neuromuscular and chest wall disorders. Cochrane Database Syst Rev 2007; (4): CD001941.

Correspondencia a:

Dr. César Maquilón O.

E-mail: cmaquilon87@hotmail.com 